\title{
Stability analysis of space-time finite integration schemes
}

\author{
$\operatorname{AUTHOR}(\mathrm{S})$ :
}

Matsuo, Tetsuji; Kawahara, Jun; Shimoi, Tomohiro; Mifune, Takeshi

\section{CITATION:}

Matsuo, Tetsuji ...[et al]. Stability analysis of space-time finite integration schemes. COMPEL - The international journal for computation and mathematics in electrical and electronic engineering 2015, 34(5): 1609-1622

\section{ISSUE DATE:}

2015-09-07

URL:

http://hdl.handle.net/2433/202624

\section{RIGHT:}

This is the version of the article that has been accepted for publication (Author Accepted Manuscript) published in final version at http://dx.doi.org/10.1108/COMPEL-02-2015-0074.; The full-text file will be made open to the public on 7 September 2016 in accordance with publisher's 'Terms and Conditions for Self-Archiving'.; This is not the published version. Please cite only the published version.; この論文は出版社版でありません。引用の際には出版社版をご磼認ご 利用ください。 


\title{
Stability Analysis of Space-Time Finite Integration Schemes
}

\author{
*Tetsuji Matsuo, *Jun Kawahara, *Tomohiro Shimoi and *Takeshi Mifune \\ *Graduate School of Engineering, Kyoto University, Kyotodaigaku-katsura, Nishikyo-ku, Kyoto 615-8510, Japan
}

\begin{abstract}
The numerical stability of a space-time finite integration (FI) method is examined. The growth rate of instability is evaluated by a numerical eigenvalue analysis formulated from the explicit time-marching scheme of the FI method. The space-time subgrid schemes using the 3D and 4D space-time FI methods are shown to be conditionally stable. For the constitutive relation at the subgrid connections, a symmetric correction is proposed that does not induce numerical instability. The staircase-type space-time subgrid improves stability compared with the straight-type subgrid.
\end{abstract}

Index Terms—eigenvalue analysis, space-time grid, subgrid, symmetric correction.

\section{INTRODUCTION}

The finite integration (FI) method (Weiland 1996) (Lager et. al 2003) (Codecasa and Politi 2008) has been reassessed for time-domain computations on unstructured spatial grids of electromagnetic fields. Similar to the FDTD method, the FI method uses a uniform time-step, which is restricted by the Courant-Friedrichs-Lewy (CFL) condition (Taflove and Hagness 2006) based on the smallest spatial grid size.

Previous work (Matsuo 2011) (Kawahara et. al 2013) introduced a space-time FI method to relax the CFL condition that achieves non-uniform time-steps on 3D and 4D space-time grids. A numerical eigenvalue analysis (Matsuo and Mifune 2014) showed that a 3D space-time FI scheme having a temporal grid subdivision can be stable. However, the eigenvalue analysis for the space-time FI scheme including both temporal and spatial grid subdivision has not yet been performed.

This study evaluates the numerical stability of $3 \mathrm{D}$ and 4D simple subgrid scheme (Matsuo et. al 2015) using the space-time FI method.

\section{SPACE-Time Finite InTEgRation Method}

\section{A. Electromagnetic Equations in Space-Time}

The coordinate system is denoted by $(c t, x, y, z)=\left(x^{0}\right.$, $\left.x^{1}, x^{2}, x^{3}\right)$, where $c=1 / \sqrt{ }\left(\varepsilon_{0} \mu_{0}\right)$ and $\varepsilon_{0}$ and $\mu_{0}$ are respectively the permittivity and permeability of the vacuum. The Maxwell equations, given in the integral form, are:

$$
\begin{aligned}
& \oint_{\partial \boldsymbol{\Omega}}=0, \oint_{\partial \underline{\underline{\Omega}} \underline{F}=\int_{\underline{\Omega}} J} \\
& F=-\sum_{i=1}^{3} E_{i} \mathrm{~d} x^{0} \mathrm{~d} x^{i}+\sum_{j=1}^{3} c B_{j} \mathrm{~d} x^{k} \mathrm{~d} x^{l} \\
& \underline{F}=\sum_{i=1}^{3} H_{i} \mathrm{~d} x^{0} \mathrm{~d} x^{i}+\sum_{j=1}^{3} c D_{j} \mathrm{~d} x^{k} \mathrm{~d} x^{l}, \\
& J=c \rho \mathrm{d} x^{1} \mathrm{~d} x^{2} \mathrm{~d} x^{3}+\sum_{j=1}^{3} J_{j} \mathrm{~d} x^{0} \mathrm{~d} x^{k} \mathrm{~d} x^{l},
\end{aligned}
$$

where $(j, k, l)$ is a cyclic permutation of $(1,2,3) ; \Omega$ and $\underline{\Omega}$ are hypersurfaces in space-time; $\rho$ is the electric charge density. In the FI method, the electromagnetic variables are defined as:

$$
f=\int_{p} F, g=\int_{\underline{\underline{p}}} \underline{F}
$$

where $p$ and $p$ are the faces of the primal and dual grids that constitute $\partial \Omega$ and $\partial \underline{\Omega}$.

To express the constitutive equation simply, the Hodge dual grid (Matsuo 2011) is introduced as

$$
\frac{\int_{\underline{p}} C_{\mathrm{r}} \mathrm{d} x^{0} \mathrm{~d} x^{j}}{\int_{p} \mathrm{~d} x^{k} \mathrm{~d} x^{l}}=-\frac{\int_{\underline{p}} \mathrm{~d} x^{k} \mathrm{~d} x^{l}}{\int_{p} c_{\mathrm{r}} \mathrm{d} x^{0} \mathrm{~d} x^{j}}=\kappa
$$

where $c_{\mathrm{r}}=1 / \sqrt{ }\left(\varepsilon_{\mathrm{r}} \mu_{\mathrm{r}}\right) ; \kappa$ is a constant determined for each pair $p$ and $\underline{p} ; \varepsilon_{\mathrm{r}}$ and $\mu_{\mathrm{r}}$ are respectively the relative permittivity and permeability. Equation (4) implies that $p$ is orthogonal to $p$ using the Lorentzian metric with speed of light $c_{\mathrm{r}} \mathrm{c}$. Combining (3) and (4) yields

$$
f=Z g / \kappa
$$

where $Z=\sqrt{ }\left(\mu_{\mathrm{r}} \mu_{0} / \varepsilon_{\mathrm{r}} \varepsilon_{0}\right)$ is the impedance.

\section{B. Explicit Time-Marching Scheme}

The FI method is generally formulated with the Maxwell grid equations using the incidence matrices from graph theory. Following (Kawahara et. al 2013), the geometrical formulation of $\left(x^{0}, x^{1}, x^{2}\right)-3 \mathrm{D}$ space-time $\mathrm{FI}$ method is summarized in Appendix $A$, where the propagation of $\left(E_{1}, E_{2}, B_{3}\right)$ is described. Equation (27) or (29) in Appendix gives an implicit expression for the time-evolution of the electromagnetic wave. There exist space-time grids having explicit time-marching schemes that are compatible with (27) (or (29)). Their numerical stability is discussed in the following sections.

\section{Stability Analysis}

Figure 1 shows an example of a space-time grid having a temporal period $\Delta x^{0}=\Delta w$, where the solid and dashed lines are the primal and dual grids, respectively. The variables given by (3) are periodically allocated along the $x^{0}$-direction on the space-time grid. The variables are accordingly denoted $\boldsymbol{V}^{0}, \boldsymbol{V}^{1}, \ldots$, where $\boldsymbol{V}^{n+1}$ is assigned after $\boldsymbol{V}^{n}$ by the time-interval $\Delta w$, as illustrated in Fig. 2 . The variable vector $\boldsymbol{V}^{n}$ is divided into $\boldsymbol{v}^{n}$ and $\boldsymbol{u}^{n}$ where the components of $\boldsymbol{v}^{n}$ are linearly independent and the components of $\boldsymbol{u}^{n}$ are given as linear combinations of the components of $\boldsymbol{v}^{n}$, i.e., $\boldsymbol{u}^{n}=\boldsymbol{P} \boldsymbol{v}^{n}$ with $\boldsymbol{P}$ a constant matrix. The numerical stability of the time-marching scheme is evaluated using the eigenvalues of matrix $\partial \boldsymbol{v}^{n+1} / \partial \boldsymbol{v}^{n}$. 
If the explicit time-marching scheme exists, its derivation from (27) takes the form

$$
\begin{aligned}
\boldsymbol{A}_{0} \boldsymbol{v}^{n} & +\boldsymbol{B}_{0} \boldsymbol{u}^{n}+\boldsymbol{A}_{1} \boldsymbol{v}^{n+1}+\boldsymbol{B}_{1} \boldsymbol{u}^{n+1} \\
& =\left(\boldsymbol{A}_{0}+\boldsymbol{B}_{0} \boldsymbol{P}\right) \boldsymbol{v}^{n}+\boldsymbol{A}_{1} \boldsymbol{v}^{n+1}+\boldsymbol{B}_{1} \boldsymbol{u}^{n+1}=0
\end{aligned}
$$

where $\boldsymbol{A}_{i}$ and $\boldsymbol{B}_{i}(i=1,2)$ are $N \times N$ and $M \times M$ constant matrices, respectively; $N$ and $M$ are the dimensions of $v$ and $\boldsymbol{u}$. The influence of permittivity/permeability of material is included in $\boldsymbol{A}_{i}$ and $\boldsymbol{B}_{i}$.

From (6), $\partial \boldsymbol{v}^{n+1} / \partial \boldsymbol{v}^{n}$ is obtained by solving

$$
\left(\begin{array}{ll}
\boldsymbol{A}_{1} & \boldsymbol{B}_{1}
\end{array}\right)\left(\begin{array}{c}
\frac{\partial \boldsymbol{v}^{n+1}}{\partial \boldsymbol{v}^{n}} \\
\frac{\partial \boldsymbol{u}^{n+1}}{\partial \boldsymbol{v}^{n}}
\end{array}\right)=-\left(\boldsymbol{A}_{0}+\boldsymbol{B}_{0} \boldsymbol{P}\right)
$$

If $\boldsymbol{u}^{n+1}$ and $\boldsymbol{v}^{n+1}$ are determined from the pairs $\boldsymbol{v}^{n}$ and $\boldsymbol{u}^{n}$ and $\boldsymbol{v}^{n}$ and $\boldsymbol{u}^{n+1}$, respectively, the time-marching scheme is rewritten as:

$$
\boldsymbol{u}^{n+1}=\boldsymbol{Q} \boldsymbol{u}^{n}+\boldsymbol{R} \boldsymbol{v}^{n}, \boldsymbol{v}^{n+1}=\boldsymbol{S} \boldsymbol{v}^{n}+\boldsymbol{T} \boldsymbol{u}^{n+1}
$$

where $\boldsymbol{Q}, \boldsymbol{R}, \boldsymbol{S}$, and $\boldsymbol{T}$ are constant matrices. Equation (8) gives

$$
\partial \boldsymbol{v}^{n+1} / \partial \boldsymbol{v}^{n}=\boldsymbol{S}+\boldsymbol{T}(\boldsymbol{R}+\boldsymbol{Q P}) .
$$

By setting

$$
\boldsymbol{A}_{0}=\left(\begin{array}{l}
\boldsymbol{S} \\
\boldsymbol{R}
\end{array}\right), \boldsymbol{B}_{0}=\left(\begin{array}{l}
\boldsymbol{O} \\
\boldsymbol{Q}
\end{array}\right), \boldsymbol{A}_{1}=\left(\begin{array}{c}
-\mathbf{1}_{N} \\
\boldsymbol{O}
\end{array}\right), \boldsymbol{B}_{1}=\left(\begin{array}{c}
\boldsymbol{T} \\
-\mathbf{1}_{M}
\end{array}\right),
$$

then (9) is equivalent to (7) because

$$
\left(\begin{array}{ll}
\boldsymbol{A}_{1} & \boldsymbol{B}_{1}
\end{array}\right)^{-1}=-\left(\begin{array}{cc}
\mathbf{1}_{N} & \boldsymbol{T} \\
\boldsymbol{O} & \mathbf{1}_{M}
\end{array}\right)
$$

where $\mathbf{1}_{N}$ and $\mathbf{1}_{M}$ are the $N \times N$ and $M \times M$ unit matrices.

Even when the material has nonlinear properties, it is possible to define $\partial \boldsymbol{v}^{n+1} / \partial \boldsymbol{v}^{n}$ and to use it for a rough evaluation of numerical stability and also for the stability analysis of the steady-state field that is static or periodic. However, the strict numerical stability analysis for general nonlinear media is difficult because $\partial \boldsymbol{v}^{n+1} / \partial \boldsymbol{v}^{n}$ depends on the filed amplitude.
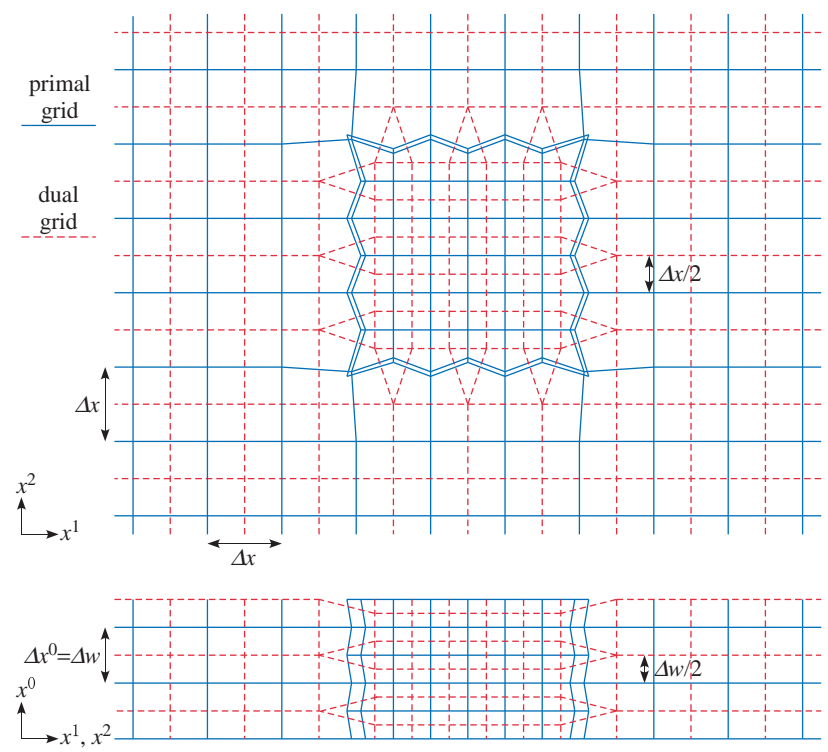

Figure 1: Space-time grid.

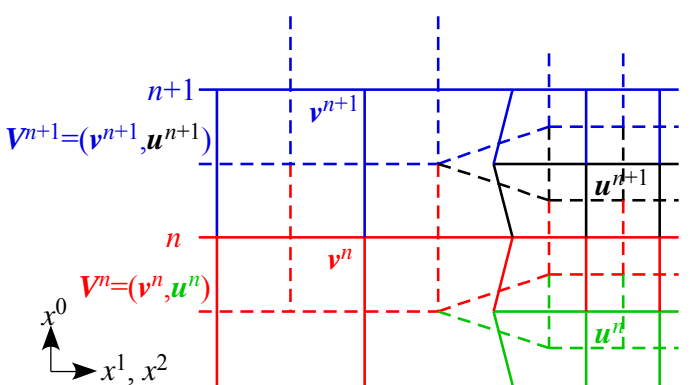

Figure 2: Periodic allocation of variables.

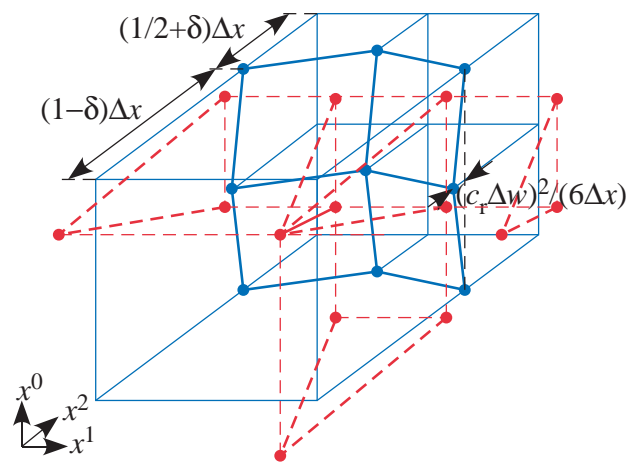

Figure 3: Connection to subgrid.

(b)

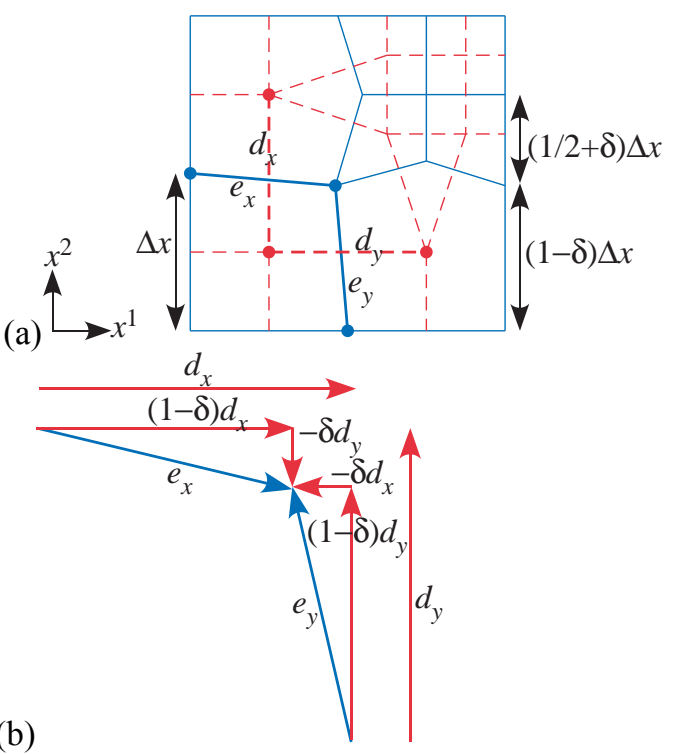

Figure 4: Corner part: (a) non-orthogonal grid, (b) symmetric correction.

\section{Computational Results}

The numerical stability of the space-time subgrid (Fig. 1) is examined. There, the spatial cell size and the temporal step of the coarse grid (main grid) are $\Delta x$ and $\Delta w=c \Delta t$, respectively, whereas those of the fine grid (subgrid) are $\Delta x / 2$ and $\Delta w / 2$.

\section{A. Stability in 3D-Space-Time}

To examine the propagation of $\left(E_{1}, E_{2}, B_{3}\right)$ on the $\left(x^{0}\right.$, $\left.x^{1}, x^{2}\right)$-3D space-time grid, its connection to the subgrid is illustrated in Fig. 3. The associated time-marching scheme is presented in (Matsuo et. al 2015).

Figure 4(a) illustrates the corner part of subgrid connection for which the variables $e_{x}$ and $e_{y}$ are given by (18) and $d_{x}$ and $d_{y}$ are given by (19); $\delta$ is a free 
parameter. When $\delta \neq 0$, the face for $e_{x}$ (or $e_{y}$ ) is not orthogonal to the edge for $d_{x}\left(d_{y}\right)$, which causes a numerical error.

Based on the vectorial relation shown in Fig. 4(b), a symmetric correction,

$$
\begin{gathered}
e_{x}=Z \Delta w\left[\left(1-\delta^{\prime}\right) d_{x}-\delta^{\prime} d_{y}\right] \\
e_{y}=Z \Delta w\left[\left(1-\delta^{\prime}\right) d_{y}-\delta^{\prime} d_{x}\right],
\end{gathered}
$$

can be used to avoid asymmetry arising in the impedance matrix. Because the face for $e_{x}$ (or $e_{y}$ ) is slanted along the $x^{0}$-direction, as in Fig. 3, $\delta$ ' is given by $\delta+\left(c_{\mathrm{r}} \Delta w\right)^{2} / 12$.

A small 3D space-time grid having spatial domain size of $30 \Delta x \times 30 \Delta x$, and including a subgrid domain of $28(\Delta x / 2) \times 28(\Delta x / 2)$, is used in the numerical eigenvalue analysis with $\varepsilon_{\mathrm{r}}=\mu_{\mathrm{r}}=1$. Spatially periodic boundary conditions are imposed.

Figure 5 plots the eigenvalue distribution of $\partial v^{n+1} / \partial v^{n}$ without correction when $\Delta w / \Delta x=0.5,0.51$ and $\delta=$ 0.08 . For $\Delta w / \Delta x=0.5$, all the eigenvalues of $\partial \boldsymbol{v}^{n+1} / \partial \boldsymbol{v}^{n}$ are on the unit circle, which implies that the explicit timemarching scheme is stable. When $\Delta w / \Delta x=0.51$, some of the eigenvalues move outside the unit circle [Fig. 5 (b)], causing numerical instability.

Figure 6 plots the eigenvalue distribution with the symmetric correction, which does not affect the stability.

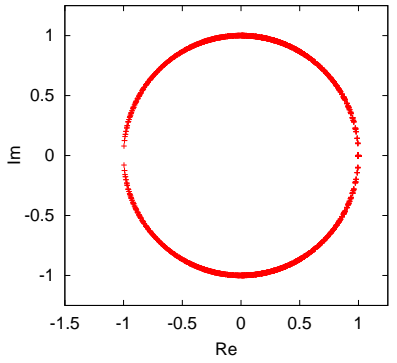

(a)

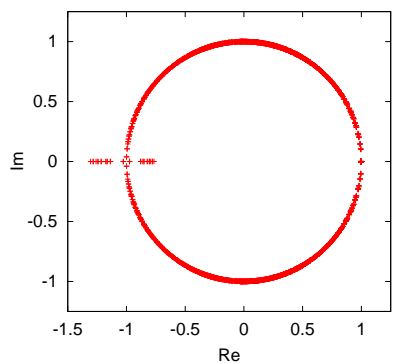

(b)
Figure 5: Eigenvalues of $\partial \boldsymbol{v}^{n+1} / \partial \boldsymbol{v}^{n}$ without correction when $\Delta w / \Delta x$ is (a) 0.5 and (b) 0.51 .

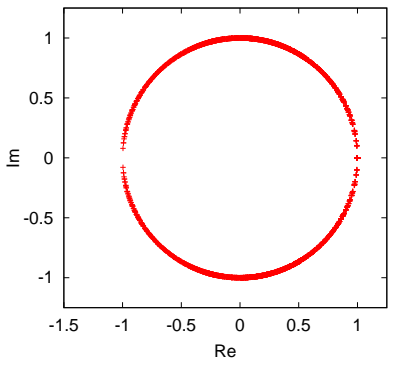

(a)

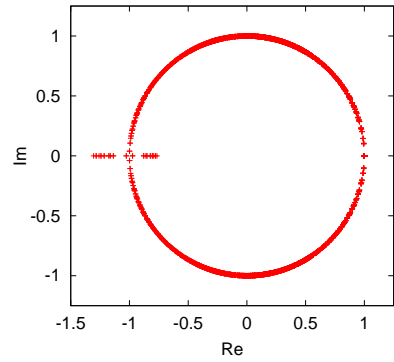

(b)
Figure 6: Eigenvalues of $\partial \boldsymbol{v}^{n+1} / \partial \boldsymbol{v}^{n}$ with symmetric correction when $\Delta w / \Delta x$ is (a) 0.5 and (b) 0.51 .

The FI method allows slanted edges to be curved [Cf. Fig. 7(a) and (b)], where node positions (•) are unchanged (Matsuo et. al 2015). A further transformation leads to staircase-like edges [Fig. 7(c)]. The edges can be transformed along the temporal direction also [Fig. 8].

Hereon, the space-time grids of Figs. 7(a) and 8(a) are referred to as straight type whereas the grids of Figs. 7(c) and $8(\mathrm{c})$ are referred to as staircase type. It is difficult for the straight type subgrid connection to locate the subgrid boundary at the planar boundary of two materials, which is possible for the staircase type connection.

Figure 9 plots the eigenvalue distribution using the staircase-type space-time grid without correction for $\Delta w /$ $\Delta x=0.5,0.51$ and $\delta=0.02$. Figure 10 plots the eigenvalue distribution with symmetric correction. Similar distributions in Figs. 9 and 10 to those in Figs. 5 and 6 show that the space-time FI method is conditionally stable.

The effect of grid type and symmetric correction on the computational accuracy is examined in Appendix B.

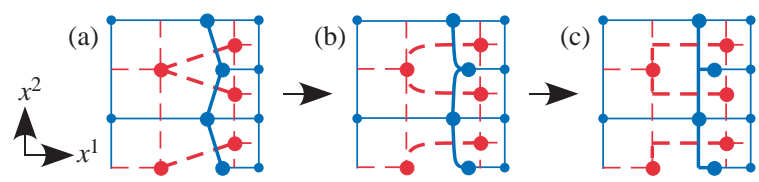

Figure 7: Transformation of spatial edges without changing node positions $(\bullet)$.

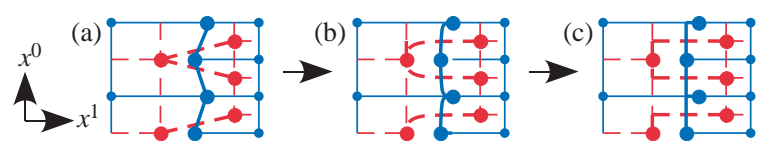

Figure 8: Transformation of space-time edges without changing node positions $(\bullet)$.

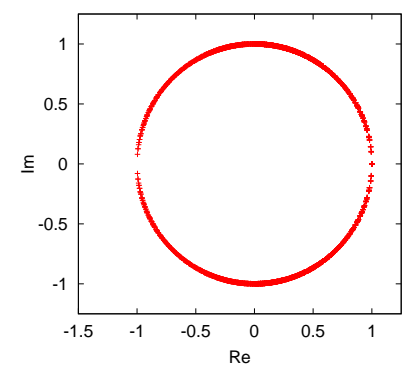

(a)

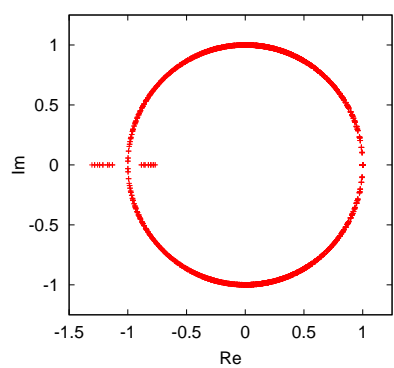

(b)
Figure 9: Eigenvalues of $\partial \boldsymbol{v}^{n+1} / \partial \boldsymbol{v}^{n}$ with staircase-type grid without correction when $\Delta w / \Delta x$ is (a) 0.5 and (b) 0.51 .

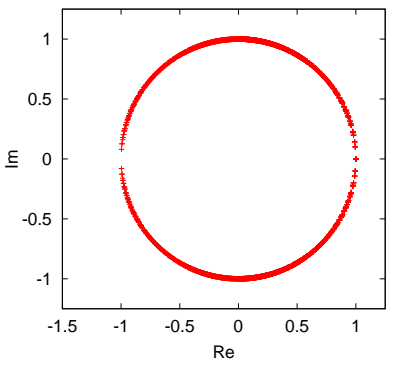

(a)

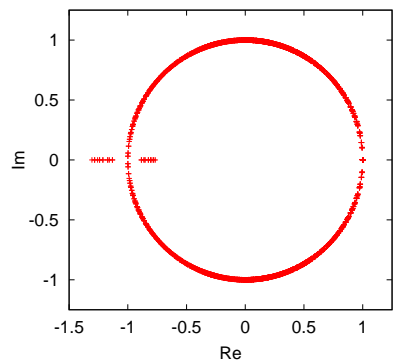

(b)
Figure 10: Eigenvalues of $\partial \boldsymbol{v}^{n+1} / \partial \boldsymbol{v}^{n}$ with staircase-type grid and symmetric correction when $\Delta w / \Delta x$ is (a) 0.5 and (b) 0.51 .

\section{B. Stability in 4D Space-Time}

Figure 11(a) illustrates a 4D connection to the subgrid of straight type, where $\delta$ is a free parameter. Similar to the case for the 3D subgrid, the edges and faces can curve resulting in a staircase-type grid [Fig. 11(b)]. 
A small 4D space-time grid having spatial domain size of $12 \Delta x \times 12 \Delta x \times 12 \Delta x$ including a subgrid domain of $8(\Delta x / 2) \times 8(\Delta x / 2) \times 8(\Delta x / 2)$ (see Fig. 12) is used in the numerical analysis of eigenvalues; spatially periodic boundary conditions are imposed. Figures 13 and 14 plot the eigenvalue distribution using the straight- and staircase-type grids, respectively. The 4D space-time FI scheme is conditionally stable because for small-valued $\Delta w / \Delta x$ all the eigenvalues of $\partial \boldsymbol{v}^{n+1} / \partial \boldsymbol{v}^{n}$ are on the unit circle. The staircase-type grid type allows a temporal step size of $\Delta w / \Delta x=0.4$ for stable explicit time marching whereas the straight-type grid suffers from instability with $\Delta w / \Delta x \geq 0.34$.
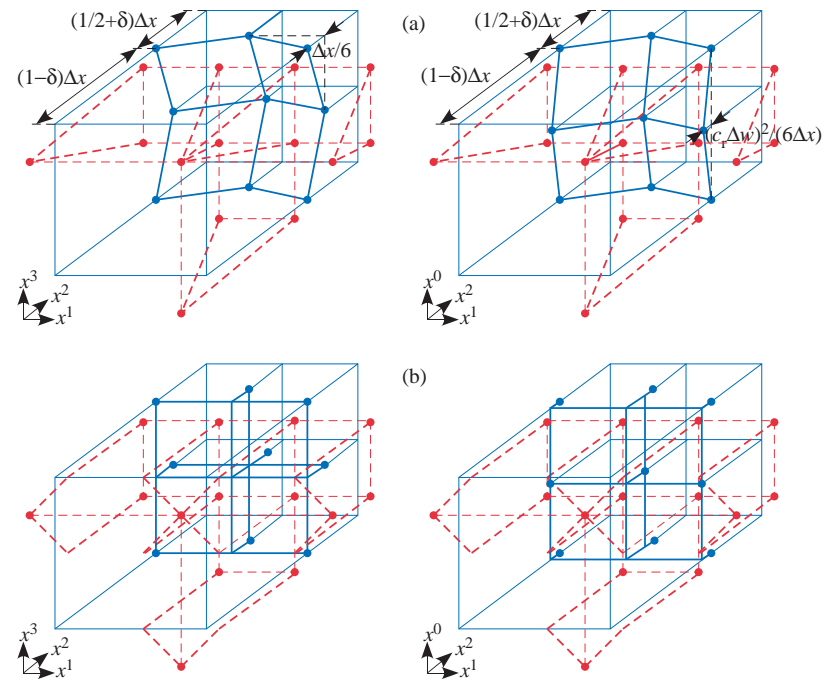

Figure 11: 4D subgrid connection of (a) straight type and (b) staircase type.

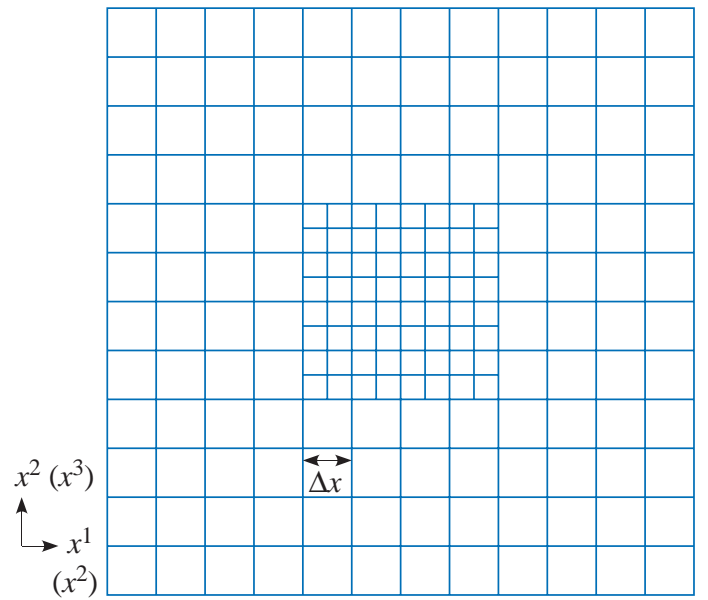

Figure 12: Small space-time grid for eigenvalue analysis.
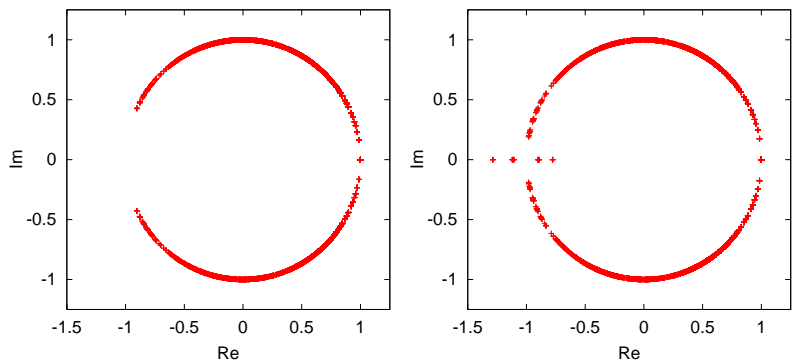

Figure 13: Eigenvalues of $\partial \boldsymbol{v}^{n+1} / \partial \boldsymbol{v}^{n}$ with 4D straight-type grid for $\Delta w / \Delta x$ is (a) 0.32 and (b) 0.34 .
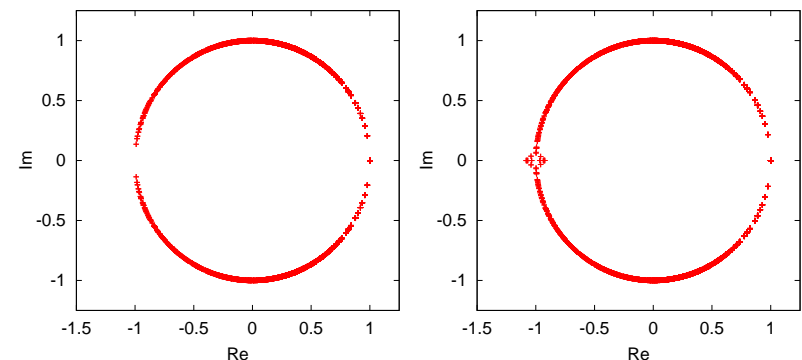

Figure 14: Eigenvalues of $\partial \boldsymbol{v}^{n+1} / \partial \boldsymbol{v}^{n}$ with 4D staircasetype grid for $\Delta w / \Delta x$ is (a) 0.4 and (b) 0.42 .

\section{V.CONCLUDING REMARKS}

A scheme for the numerical analysis of stability of the space-time FI method is presented. The 3D and 4D subgrid schemes using the space-time FI method are conditionally stable, where a symmetric correction for the constitutive relation does not induce numerical instability. The staircase-type 4D space-time subgrid allows a larger time-step than the straight-type subgrid.

\section{APPENDIX}

\section{A. Geometrical Formulation of Space-Time FI method}

The geometrical formulation of the 3D space-time FI method is derived, where the coordinates are $\left(x^{0}, x^{1}, x^{2}\right)$ and the propagation of fields $\left(E_{1}, E_{2}, B_{3}\right)$ is described:

$$
\begin{aligned}
& F=c B_{3} \mathrm{~d} x^{1} \mathrm{~d} x^{2}+E_{2} \mathrm{~d} x^{2} \mathrm{~d} x^{0}-E_{1} \mathrm{~d} x^{0} \mathrm{~d} x^{1}, \\
& \underline{F}=H_{3} \mathrm{~d} x^{0}-c D_{2} \mathrm{~d} x^{1}+c D_{1} \mathrm{~d} x^{2} .
\end{aligned}
$$

Compared with the $4 \mathrm{D}$ case, $\underline{F} \mathrm{~d} x^{3}$ corresponds to $\underline{F}$ in (2).

Let arrays $\{n\},\{s\},\{p\}$, and $\{v\}$ denote the sets of nodes, edges, faces, and volumes in the $3 \mathrm{D}$ space-time primal grid, respectively. These are related by incidence matrices $[G],[C]$, and $[D]$ as.

$$
\partial\{s\}=[G]\{n\}, \partial\{p\}=[C]\{s\}, \partial\{v\}=[D]\{p\}
$$

where $\partial$ denotes the restriction to the boundary. Similarly, the sets of nodes, edges, faces, and volumes in the dual grid are related as,

$$
\partial\{\underline{s}\}=[\underline{G}]\{\underline{n}\}, \partial\{\underline{p}\}=[\underline{C}]\{\underline{s}\}, \partial\{\underline{v}\}=[\underline{D}]\{\underline{p}\}
$$

The $k$-th face $p_{(k)}$ on the primal grid and its corresponding edge $\underline{S}_{(k)}$ on the dual grid are written

$$
\begin{aligned}
& p_{(k)}=p_{(k)}^{0} \mathrm{~d} x_{1} \mathrm{~d} x_{2}+p_{(k)}^{1} \mathrm{~d} x_{2} \mathrm{~d} x_{0}+p_{(k)}{ }^{2} \mathrm{~d} x_{0} \mathrm{~d} x_{1} \\
& \underline{S}_{(k)}=\underline{s}_{(k)}{ }^{0} \mathrm{~d} x_{0}+\underline{s}_{(k)}^{1} \mathrm{~d} x_{1}+\underline{s}_{(k)}{ }^{2} \mathrm{~d} x_{2} .
\end{aligned}
$$

Using $p_{(k)}{ }^{i}$ and $\underline{s}_{(k)}^{i}$, the area vector $\boldsymbol{p}_{(k)}$ and the edge vector $\underline{\boldsymbol{S}}_{(k)}$ are defined as

$$
\boldsymbol{p}_{(k)}=\left(p_{(k)}{ }^{0}, p_{(k)}{ }^{1}, p_{(k)}{ }^{2}\right), \underline{\boldsymbol{s}}_{(k)}=\left(\underline{s}_{(k)}{ }^{0}, \underline{s}_{(k)}{ }^{1}, \underline{S}_{(k)}{ }^{2}\right)
$$

Corresponding to $p_{(k)}$ and $\underline{s}_{(k)}$, the variables for the space-time FI method is given as

$$
\begin{gathered}
f_{(k)}=\int_{p(k)} F=p_{(k)}{ }^{0} c B_{3[\mathrm{k}]}+p_{(k)}{ }^{1} E_{2[\mathrm{k}]}-p_{(k)}{ }^{2} E_{1[\mathrm{k}]}, \\
g_{(k)}=\int_{\underline{s}(k)} \underline{F}=\underline{s}_{(k)}{ }^{0} H_{3[\mathrm{k}]}-\underline{s}_{(k)}{ }^{1} c D_{2[\mathrm{k}]}+\underline{s}_{(k)}{ }^{2} c D_{1[\mathrm{k}]},
\end{gathered}
$$


where the subscript ' $[k]$ ' denotes an averaged value on $p_{(k)}$ or $\underline{s}_{(k)}$. Equation (4) is rewritten as

$$
c_{\mathrm{r}} \underline{\underline{S}}_{(k)}^{0} / p_{(k)}{ }^{0}=-\underline{\boldsymbol{s}}_{(k)}{ }^{1} / c_{\mathrm{r}} p_{(k)}{ }^{1}=-\underline{\boldsymbol{s}}_{(k)}{ }^{2} / c_{\mathrm{r}} p_{(k)}{ }^{2}=\kappa_{(k)},
$$

and implies that $\underline{s}_{(k)}$ is orthogonal to $p_{(k)}$ by the Lorentzian metric but may be non-orthogonal to $p_{(k)}$ by the Euclidean metric. Figure 15 illustrates the geometrical relation between $p_{(k)}$ and $\underline{s}_{(k)}$, where $\boldsymbol{p}_{(k)} \underline{\mathbf{s}}_{(k)}$ denotes the inner product using the Euclidean metric.

From (18)-(20), $f_{(k)}$ and $g_{(k)}$ are related by impedance $Z_{(k)}$ :

$$
f_{(k)}=z_{(k)} g_{(k)}
$$

where

$$
Z_{(k)}=Z_{(k)} / \kappa .
$$

In integral form, Maxwell's equations (1) without source terms are

$$
[D]\{f\}=0,[\underline{C}]\{g\}=0,
$$

where $\{f\}$ and $\{\mathrm{g}$ \} consist of the variables defined by (18) and (19). In matrix form,

$$
\{f\}=[z]\{g\},
$$

where $[z]$ is the diagonal matrix formed with elements (22).
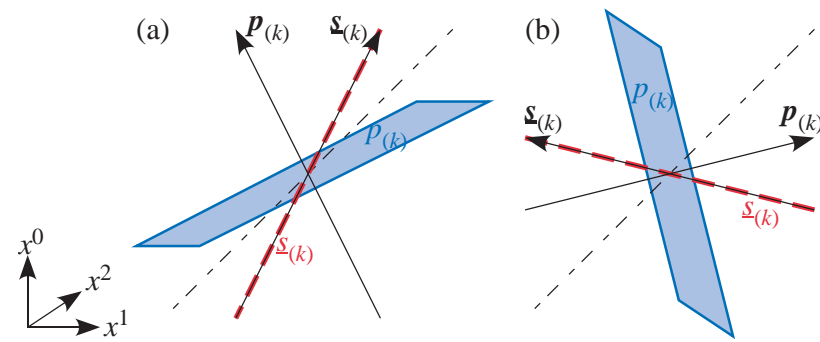

Figure 15: Geometrical relation between face $p_{(k)}$ and edge $\underline{\boldsymbol{s}}_{(k)}:$ (a) $\boldsymbol{p}_{(k)} \underline{\mathbf{s}}_{(k)}>0$, and (b) $\boldsymbol{p}_{(k)} \underline{\mathbf{s}}_{(k)}<0$.

If vectors $p_{(k)}$ and $\underline{s}_{(k)}$ are represented as in Fig. 15, $\underline{s}_{(k)}$ may have the opposite direction [Fig. 15(b)]. This fact is due to the relation (20) and results in the relation

$$
[\underline{C}]=[C]^{* T} \text {. }
$$

The matrix operator ${ }^{*}$ is defined as

$$
\underline{C}_{i j}=\left\{\begin{array}{cc}
c_{j i} & \left(c_{j i} \neq 0, \boldsymbol{p}_{(k)} \cdot \underline{\boldsymbol{s}}_{(k)}>0\right) \\
-c_{j i} & \left(c_{j i} \neq 0, \boldsymbol{p}_{(k)} \cdot \underline{\boldsymbol{s}}_{(k)}<0\right) \\
0 & \left(c_{j i}=0\right)
\end{array}\right.
$$

where $c_{i j}$ and $\underline{c}_{j i}$ are the respective elements of $[C]$ and $[\underline{C}]$ corresponding to the pair $p_{(k)}$ and $\underline{s}_{(k)}$.

Equations (22), (23), and (25) determine the space-time Maxwell grid equations

$$
\left(\begin{array}{c}
{[D]} \\
{[C]^{* \mathrm{~T}}[z]^{-1}}
\end{array}\right)\{f\}=0 .
$$

By modifying the impedance matrix, another formulation is possible, where relation $[\underline{C}]=[C]^{\mathrm{T}}$ holds. The modified impedance matrix $\left[z^{*}\right]$ is defined as

$$
z_{(k)}^{*}=\left\{\begin{array}{cc}
z_{(k)} & \left(\boldsymbol{p}_{(k)} \cdot \underline{\boldsymbol{s}}_{(k)}>0\right) \\
-z_{(k)} & \left(\boldsymbol{p}_{(k)} \cdot \underline{\boldsymbol{s}}_{(k)}<0\right)
\end{array}\right.
$$

where $z_{(k)}{ }^{*}$ is the element of $[z]$ corresponding to the pair $p_{(k)}$ and $\underline{s}_{(k)}$. Equations (23), (24), and (28) determine another form of the space-time Maxwell grid equations,

$$
\left(\begin{array}{c}
{[D]} \\
{[C]^{\mathrm{T}}\left[z^{*}\right]^{-1}}
\end{array}\right)\{f\}=0,
$$

which is equivalent to (27).

\section{B. Effect of Symmetrical Correction}

Wave propagation is simulated to compare subgrid schemes in the same way as in (Matsuo et. al 2015). For simplicity, the permittivity and permeability are set to unity by normalization; also $\Delta x$ and $\Delta w$ are set to 1 and 0.5 by normalization. The spatial domain of [-80: 80$] \times$ [-80: 80] includes a subgrid domain of [16:36] $\times[20$ : 40] having spatial step $\Delta x / 2$ and temporal step $\Delta w / 2$. The normalized initial conditions are $E_{1}=E_{2}=0$ and $B_{3}=$ $\exp \left\{-\left[\left(x^{1}\right)^{2}+\left(x^{2}\right)^{2}\right] / 25\right\}$. Spatially periodic boundary conditions are imposed. Figure 16 presents the distributions of discrepancy $\Delta B$ between $B_{3}$ obtained by the FDTD method and the space-time subgrid at $c t=60$. The discrepancy seen for $x \geq 16$ and $y \geq 20$ is mainly caused by numerical dispersion whereas that for $x \leq 16$ or $y \leq 20$ is caused by an unphysical wave reflection at the subgrid connection. The staircase-type grid with symmetric correction reduces this unphysical reflection.

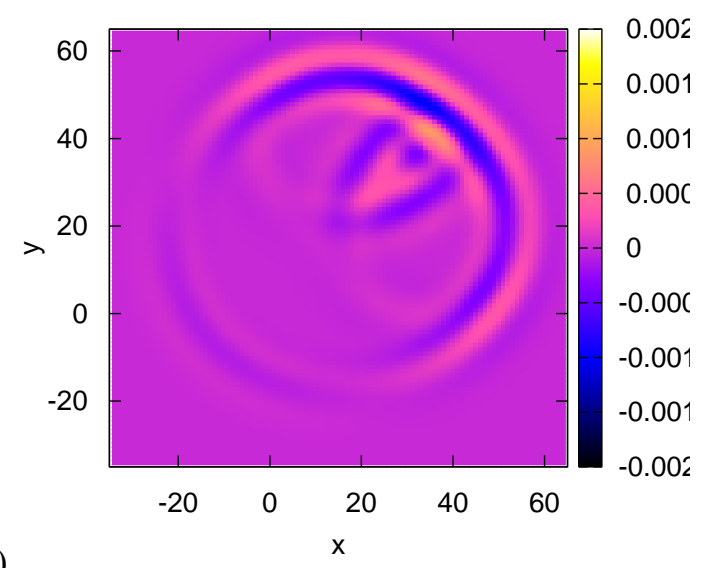

(a)

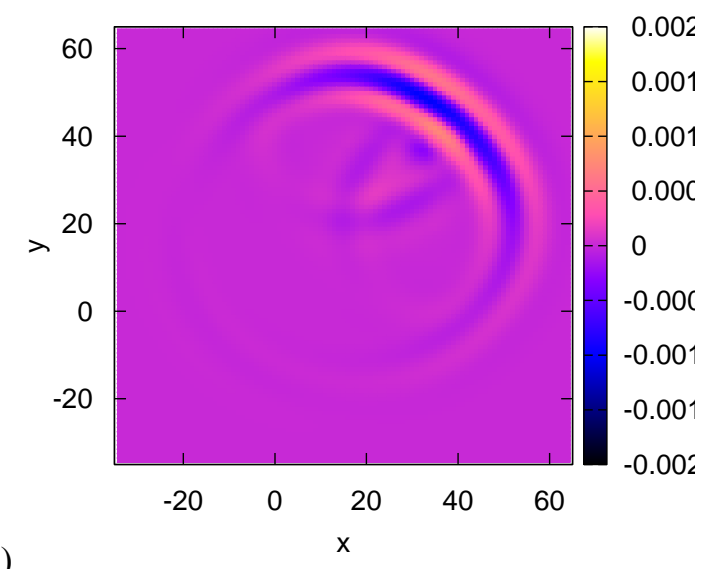

Figure 16: Discrepancy $\Delta B$ relative to $B_{3}$ obtained employing the FDTD method: (a) straight-type grid without correction, and (b) staircase-type grid with symmetric correction. 


\section{REFERENCES}

Codecasa, L. and Politi, M. (2008), "Explicit, consistent, and conditionally stable extension of FD-TD to tetrahedral grids by FIT," IEEE Trans. Magn., Vol. 44, No. 6, pp. 1258-1261.

Kawahara, J., Mifune, T. and Matsuo, T. (2013), "Geometrical formulation of a 3D space-time finite integration method," IEEE Trans. Magn., Vol. 49, No. 5, pp. 1693-1696.

Lager, I. E., Tonti, E., de Hoop, A.T., Mur, G. and Marrone, M. (2003),

"Finite formulation and domain-integrated field relations in electromagnetics - a synthesis," IEEE Trans. Magn., Vol. 39, No. 3, pp. 1199-1202.

Matsuo, T. (2011), "Space-Time finite integration method for electromagnetic field computation,” IEEE Trans. Magn., Vol. 47, No. 5, pp. 1530-1533.

Matsuo, T. and Mifune, T. (2014), "Reduction of unphysical wave reflection arising from space-time finite integration method," IEEE Trans. Magn., Vol. 50, No. 2, 7004204.

Matsuo, T., Shimoi, T., Kawahara, J. and Mifune, T (2015), “A simple subgrid scheme using space-time finite integration method," IEEE Trans. Magn., Vol. 51, No. 3, 7201904.

Taflove, A. and Hagness, S. C. (2005), Computational Electromagnetics, The Finite Difference in Time Domain Method, $3^{\text {rd }} \mathrm{Ed}$. Boston, MA: Artech House.

Weiland, T. (1996), "Time domain electromagnetic field computation with finite difference methods," Int. J. Numer. Model, Vol. 9, No. 4, pp. 295-319. 